\title{
Orbit inequivalent actions for groups containing a copy of $\mathbb{F}_{2}$
}

\author{
Adrian Ioana
}

Received: 4 June 2008 / Accepted: 24 November 2010 / Published online: 7 December 2010 (C) The Author(s) 2010. This article is published with open access at Springerlink.com

\begin{abstract}
We prove that if a countable group $\Gamma$ contains a copy of $\mathbb{F}_{2}$, then it admits uncountably many non orbit equivalent actions.
\end{abstract}

\section{O Introduction}

Throughout this paper we consider free, ergodic, measure preserving (m.p.) actions $\Gamma \curvearrowright(X, \mu)$ of countable, discrete groups $\Gamma$ on standard probability spaces $(X, \mu)$. Measurable group theory is roughly the study of such group actions from the viewpoint of the induced orbit equivalence relation. A basic question in measurable group theory is to find groups $\Gamma$ which admit many non-orbit equivalent actions (see the survey [35]). In this respect, recall that two free, ergodic, m.p. actions $\Gamma \curvearrowright(X, \mu)$ and $\Lambda \curvearrowright(Y, v)$ are said to be orbit equivalent $(O E)$ if they induce isomorphic equivalence relations, i.e. if there exists a measure space isomorphism $\theta:(X, \mu) \rightarrow(Y, v)$ such that $\theta(\Gamma x)=\Lambda \theta(x)$, for almost every $x \in X$.

The striking lack of rigidity manifested by amenable groups (any two free, ergodic m.p. actions of any two infinite amenable groups $\Gamma$ and $\Lambda$ are orbit equivalent-a result proved by Dye in the case $\Gamma$ and $\Lambda$ are Abelian [8] and by Ornstein-Weiss in general ([29], see also [6])) implies that the above question is well-posed only for non-amenable groups. For a non-amenable group $\Gamma$, it is known that $\Gamma$ admits at least two non- $O E$ actions $[5,18,36]$. Moreover, recently, the following classes of non-amenable groups have been shown to admit uncountably many non- $O E$ actions: property (T) groups [18], free groups

\footnotetext{
A. Ioana ( $\triangle)$

Department of Mathematics, CALTECH, Pasadena, CA 91125, USA

e-mail: aioana@caltech.edu
} 
([17], see also [20, 38]), weakly rigid groups [31], non-amenable products of infinite groups ([33], see also [19,26]) and mapping class groups [23]. These classes of groups added to the already known ones [1, 15, 39].

In this paper, we prove that the same is true for a new, large class of nonamenable groups.

Main Result Let $\Gamma$ be a countable discrete group which contains a copy of the free group $\mathbb{F}_{2}$. Then $\Gamma$ has uncountably many non-OE actions.

Note that this result covers most non-amenable groups. The question of whether every non-amenable $\Gamma$ contains a copy of $\mathbb{F}_{2}$, known as von Neumann's problem, was open for a long time, until it was settled in the negative by Ol'shanskii [28].

Remark In Sect. 3, we prove moreover that the main result remains true under the weaker assumption that $\Gamma$ is measure equivalent to a group containing a copy of $\mathbb{F}_{2}$. Subsequently, a combination of results, ideas from $[9,16]$ and the present paper has been used to show that the above result holds true for any non-amenable group $\Gamma$ [9]. This development led us to several applications:

Corollary 1 A countable group $\Gamma$ is non-amenable if and only if we can find a free, ergodic, m.p. action $\Gamma \curvearrowright(X, \mu)$ and a von Neumann subalgebra $Q \subset L^{\infty}(X, \mu)$ such that

- $Q^{\prime} \cap L^{\infty}(X, \mu) \rtimes \Gamma=L^{\infty}(X, \mu)$ and

- the inclusion $Q \subset L^{\infty}(X, \mu) \rtimes \Gamma$ has relative property $(T)$ (in the sense of [30]).

Corollary 2 A countable group $\Gamma$ is $H T$ (in the sense of [30]) if and only if is non-amenable and has Haagerup's property.

Corollary 3 Any countable, non-amenable group $\Gamma$ admits continuum many non-von Neumann equivalent actions.

For the proofs and the definitions of the notions involved above we refer the reader to Sect. 4. Note that Corollary 3 strengthens the main result of [9].

To outline the proof of the main result, recall that if we view $\mathbb{F}_{2}$ as a finite index subgroup of $\mathrm{SL}_{2}(\mathbb{Z})$, then the pair $\left(\mathbb{F}_{2} \ltimes \mathbb{Z}^{2}, \mathbb{Z}^{2}\right)$ has the relative property (T) of Kazhdan-Margulis [21, 25]. This fact implies that the induced m.p. action $\mathbb{F}_{2} \curvearrowright^{\alpha} \mathbb{T}^{2}=\hat{\mathbb{Z}}^{2}$ is rigid, in the sense of Popa [30]. The main idea of the proof is then to consider the class $\mathcal{F}$ of actions $\Gamma \curvearrowright X$ for which the restriction $\mathbb{F}_{2} \curvearrowright X$ admits $\alpha$ as a quotient. Note that the rigidity of $\alpha$ has been successfully used before by Gaboriau and Popa to show that non-Abelian free groups admit continuum many non-OE actions [17]. 
Using a separability argument (in the spirit of $[4,17,30]$ ) in connection with the rigidity of $\alpha$, we prove that in every uncountable set $\mathcal{S} \subset \mathcal{F}$ consisting of mutually orbit equivalent actions we can find two actions whose restrictions to $\mathbb{F}_{2}$ are conjugate. On the other hand, using the co-induced construction (see Sect. 2) we provide continuum many actions in $\mathcal{F}$ for which the restrictions to $\mathbb{F}_{2}$ are mutually non-conjugate. Altogether, we deduce that continuum many actions from $\mathcal{F}$ are non-orbit equivalent.

\section{A separability argument}

\subsection{Conventions}

We start this section by recalling some of the notions that we will use further. For this, fix two m.p actions $\Gamma \curvearrowright^{\sigma}(X, \mu)$ and $\Gamma \curvearrowright^{\alpha}(Z, v)$ of a countable group $\Gamma$.

(i) The unitary representation $\pi_{\sigma}: \Gamma \rightarrow \mathcal{U}\left(\left(L^{2}(X, \mu)\right)\right.$ induced by $\sigma$ is defined by $\pi_{\sigma}(\gamma)(f)=f \circ \sigma\left(\gamma^{-1}\right)$, for all $f \in L^{2}(X, \mu)$ and $\gamma \in \Gamma$. We denote by $\pi_{\sigma}^{0}$ the restriction of $\pi_{\sigma}$ to $L^{2}(X, \mu) \ominus \mathbb{C} 1$.

(ii) If $Y \subset X$ is a measurable $\sigma(\Gamma)$-invariant set, then we call the action $\Gamma \curvearrowright^{\sigma}\left(Y, \frac{\mu_{\mid Y}}{\mu(Y)}\right)$ the restriction of $\sigma$ to $Y$ and we denote it $\sigma_{\mid Y}$. In this case, we have that $\pi_{\sigma}=\pi_{\sigma_{\mid Y}} \oplus \pi_{\sigma_{\mid X \backslash Y}}$.

(iii) We say that $\alpha$ is a quotient of $\sigma$ if there exists a measurable, measure preserving, onto map $p: X \rightarrow Z$ (called the quotient map) such that $p \circ \sigma(\gamma)=\alpha(\gamma) \circ p$, for all $\gamma \in \Gamma$. In this case, we have that $\pi_{\alpha} \subset \pi_{\sigma}$.

(iv) We say that $\alpha$ and $\sigma$ are conjugate if there exists a measure space isomorphism $p: X \rightarrow Z$ satisfying the condition in (iii). In this case, we have that $\pi_{\alpha}=\pi_{\sigma}$.

(v) The diagonal product of $\alpha$ and $\sigma$ is the action of $\Gamma$ on $(Z, \nu) \times(X, \mu)$ given by $(\alpha \times \sigma)(\gamma)=\alpha(\gamma) \times \sigma(\gamma)$ for all $\gamma \in \Gamma$.

\subsection{Relative property $(\mathrm{T})$}

For an inclusion $\Gamma_{0} \subset \Gamma$ of countable, discrete groups we say that the pair $\left(\Gamma, \Gamma_{0}\right)$ has relative property $(\mathrm{T})$ if for all $\varepsilon>0$, there exists $\delta>0$ and $F \subset \Gamma$ finite such that if $\pi: \Gamma \rightarrow \mathcal{U}(\mathcal{H})$ is a unitary representation and $\xi \in \mathcal{H}$ is a unit vector satisfying

$$
\|\pi(g)(\xi)-\xi\|<\delta, \quad \forall g \in F,
$$

then there exists $\xi_{0} \in \mathcal{H}$ such that

$$
\left\|\xi_{0}-\xi\right\|<\varepsilon, \quad \pi(h)\left(\xi_{0}\right)=\xi_{0}, \quad \forall h \in \Gamma_{0} .
$$


Following Kazhdan-Margulis, the pair $\left(\mathrm{SL}_{2}(\mathbb{Z}) \ltimes \mathbb{Z}^{2}, \mathbb{Z}^{2}\right)$, where $\mathrm{SL}_{2}(\mathbb{Z})$ acts on $\mathbb{Z}^{2}$ by matrix multiplication, has relative property $(T)[21,25]$. In fact, for any non-amenable subgroup $\Gamma$ of $\mathrm{SL}_{2}(\mathbb{Z})$, the pair $\left(\Gamma \ltimes \mathbb{Z}^{2}, \mathbb{Z}^{2}\right)$ has relative property (T) [2]. For more examples of pairs of groups with relative property $(\mathrm{T})$, see $[10,34]$.

From now on, we fix a countable group $\Gamma$ which contains a copy of $\mathbb{F}_{2}$. We also fix a free subgroup $\mathbb{F}_{2} \subset \Gamma$. Next, we view $\mathbb{F}_{2}$ as a finite index subgroup of $\mathrm{SL}_{2}(\mathbb{Z})$. In particular, we get that the pair $\left(\mathbb{F}_{2} \ltimes \mathbb{Z}^{2}, \mathbb{Z}^{2}\right)$ has relative property $(\mathrm{T})$. Also, we denote by $\alpha$ the action of $\mathbb{F}_{2}$ on $\mathbb{T}^{2}=\hat{\mathbb{Z}}^{2}$ induced by the action of $\mathbb{F}_{2}$ on $\mathbb{Z}^{2}$. Note that this action preserves the Haar measure $\lambda^{2}$ of $\mathbb{T}^{2}$ and is free and weakly mixing. Finally, we represent the group $\mathbb{F}_{2} \ltimes \mathbb{Z}^{2}$ as $\left\{(a, \gamma) \mid a \in \mathbb{Z}^{2}, \gamma \in \mathbb{F}_{2}\right\}$ with the group multiplication given by $\left(a_{1}, \gamma_{1}\right) \circ\left(a_{2}, \gamma_{2}\right)=\left(a_{1} \gamma_{1}\left(a_{2}\right), \gamma_{1} \gamma_{2}\right)$.

Theorem 1.3 Let $\mathcal{F}$ be the class of free, ergodic, m.p. actions $\Gamma \curvearrowright^{\sigma}(X, \mu)$ on a fixed standard probability space $(X, \mu)$ satisfying the following:

(i) $\alpha$ is a quotient of $\sigma_{\mid \mathbb{F}_{2}}$, with the quotient map $p_{\sigma}: X \rightarrow \mathbb{T}^{2}$.

(ii) $\forall \gamma \in \Gamma \backslash\{e\}$, the set $\left\{x \in X \mid p_{\sigma}(\gamma x)=p_{\sigma}(x)\right\}$ has zero measure.

Let $\left\{\sigma_{i}\right\}_{i \in I} \subset \mathcal{F}$ be an uncountable family of mutually orbit equivalent actions. Then there exists an uncountable set $J \subset I$ with the following property: for every $i, j \in J$, there exist two measurable sets $X_{i}, X_{j} \subset X$ of positive measure such that $X_{i}$ is $\sigma_{i}\left(\mathbb{F}_{2}\right)$-invariant, $X_{j}$ is $\sigma_{j}\left(\mathbb{F}_{2}\right)$-invariant and the restriction of $\sigma_{i \mid \mathbb{F}_{2}}$ to $X_{i}$ is conjugate to the restriction of $\sigma_{j \mid \mathbb{F}_{2}}$ to $X_{j}$.

Proof Using the hypothesis we can actually assume that all $\sigma_{i}$ generate the same measurable equivalence relation $\mathcal{R} \subset X \times X$, i.e.

$$
\mathcal{R}=\left\{\left(x, \sigma_{i}(\gamma)(x)\right) \mid x \in X, \gamma \in \Gamma\right\}, \quad \forall i \in I .
$$

Following [11], we endow $\mathcal{R}$ with the measure $\tilde{\mu}$ given by

$$
\tilde{\mu}(A)=\int_{X}|A \cap(\{x\} \times X)| d \mu(x),
$$

for every Borel subset $A \subset \mathcal{R}$.

By condition (i), for every $j \in I$, we can find a quotient map $p_{j}:(X, \mu) \rightarrow$ $\left(\mathbb{T}^{2}, \lambda^{2}\right)$ a such that $p_{j} \circ \sigma_{j}(\gamma)=\alpha(\gamma) \circ p_{j}$, for all $\gamma \in \mathbb{F}_{2}$. If $a \in \mathbb{Z}^{2}$, then we view $a$ as a character on $\mathbb{T}^{2}$ and we define $\eta_{a}^{j}=a \circ p_{j} \in L^{\infty}(X, \mu)$, for all $j \in I$. It is easy to see that for all $a \in \mathbb{Z}^{2}, \gamma \in \mathbb{F}_{2}$ and $j \in I$ we have that $\eta_{\gamma(a)}^{j}=\eta_{a}^{j} \circ \sigma_{j}\left(\gamma^{-1}\right)$. Using this relation it follows that the formula

$$
\pi_{i, j}(a, \gamma)(f)(x, y)=\eta_{a}^{i}(x) \overline{\eta_{a}^{j}(y)} f\left(\sigma_{i}\left(\gamma^{-1}\right)(x), \sigma_{j}\left(\gamma^{-1}\right)(y)\right),
$$


for all $f \in L^{2}(\mathcal{R}, \tilde{\mu}),(x, y) \in \mathcal{R}$ and $(a, \gamma) \in \mathbb{F}_{2} \ltimes \mathbb{Z}^{2}$, defines a unitary representation $\pi_{i, j}: \mathbb{F}_{2} \ltimes \mathbb{Z}^{2} \rightarrow \mathcal{U}\left(L^{2}(\mathcal{R}, \tilde{\mu})\right)$, for every $i, j \in I$.

Let $\xi=1_{\Delta}$, where $\Delta=\{(x, x), x \in X\}$, then $\xi \in L^{2}(\mathcal{R}, \tilde{\mu})$ and $\|\xi\|_{L^{2}(\mathcal{R}, \tilde{\mu})}=1$. Given two functions $\phi_{1}, \phi_{2}: X \rightarrow X$, we denote by $1_{\left\{\phi_{1}=\phi_{2}\right\}}$ the characteristic function of the set of all $x \in X$ such that $\phi_{1}(x)=\phi_{2}(x)$. For every $i, j \in I$ and all $(a, \gamma) \in \mathbb{F}_{2} \ltimes \mathbb{Z}^{2}$ we have that

$$
\begin{aligned}
& \left\|\pi_{i, j}(a, \gamma)(\xi)-\xi\right\|_{L^{2}(\mathcal{R}, \tilde{\mu})}^{2} \\
& =2-2 \Re\left\langle\pi_{i, j}(a, \gamma)(\xi), \xi\right\rangle_{L^{2}(\mathcal{R}, \tilde{\mu})} \\
& =2-2 \Re \int_{X} \eta_{a}^{i}(x) \overline{\eta_{a}^{j}(x)} 1_{\left\{\sigma_{i}\left(\gamma^{-1}\right)=\sigma_{j}\left(\gamma^{-1}\right)\right\}}(x) d \mu(x) \\
& \left.\quad \text { if }\|f\|_{\infty},\|g\|_{\infty} \leq 1, \text { then } \Re \int_{X}(1-f g) \leq\|1-f\|_{2}+\|1-g\|_{2}\right) \\
& \leq 2\left\|1-1_{\left\{\sigma_{i}\left(\gamma^{-1}\right)=\sigma_{j}\left(\gamma^{-1}\right)\right\}}\right\|_{L^{2}(X, \mu)}+2\left\|1-\eta_{a}^{i} \overline{\eta_{a}^{j}}\right\|_{L^{2}(X, \mu)} \\
& =2\left\|1_{\left\{\left(x, \sigma_{i}\left(\gamma^{-1}\right)(x)\right) \mid x \in X\right\}}-1_{\left\{\left(x, \sigma_{j}\left(\gamma^{-1}\right)(x)\right) \mid x \in X\right\}}\right\|_{L^{2}(\mathcal{R}, \tilde{\mu})} \\
& \quad+2\left\|\eta_{a}^{i} 1_{\Delta}-\eta_{a}^{j} 1_{\Delta}\right\|_{L^{2}(\mathcal{R}, \tilde{\mu})} .
\end{aligned}
$$

Now, since the pair $\left(\mathbb{F}_{2} \ltimes \mathbb{Z}^{2}, \mathbb{Z}^{2}\right)$ has relative property (T), we can find $\delta>0$ and $A \subset \mathbb{Z}^{2}, B \subset \mathbb{F}_{2}$ finite sets such that if $\pi: \mathbb{F}_{2} \ltimes \mathbb{Z}^{2} \rightarrow \mathcal{U}(\mathcal{H})$ is a unitary representation and $\xi \in \mathcal{H}$ is a unit vector which satisfies

$$
\|\pi(a, \gamma)(\xi)-\xi\| \leq \delta, \quad \forall(a, \gamma) \in A \times B,
$$

then there exists a $\pi\left(\mathbb{Z}^{2}\right)$-invariant vector $\xi_{0} \in \mathcal{H}$ such that $\left\|\xi_{0}-\xi\right\| \leq 1 / 2$.

Next, since the Hilbert space $L^{2}(\mathcal{R}, \tilde{\mu})$ is separable and $I$ is uncountable, we can find $J \subset I$ uncountable such that

$$
\left\|\eta_{a}^{i} 1_{\Delta}-\eta_{a}^{j} 1_{\Delta}\right\|_{L^{2}(\mathcal{R}, \tilde{\mu})} \leq \delta^{2} / 4, \quad \forall a \in A
$$

and

$$
\left\|1_{\left\{\left(x, \sigma_{i}\left(\gamma^{-1}\right)(x)\right) \mid x \in X\right\}}-1_{\left\{\left(x, \sigma_{j}\left(\gamma^{-1}\right)(x)\right) \mid x \in X\right\}}\right\|_{L^{2}(\mathcal{R}, \tilde{\mu})} \leq \delta^{2} / 4, \quad \forall \gamma \in B,
$$

for all $i, j \in J$. When combined with inequality (1), this gives that

$$
\left\|\pi_{i, j}(a, \gamma)(\xi)-\xi\right\|_{L^{2}(\mathcal{R}, \tilde{\mu})} \leq \delta, \quad \forall(a, \gamma) \in A \times B, \quad \forall i, j \in J
$$

Fix $i, j \in J$. Then by using relative property (T) together with (2) we can find $f \in L^{2}(\mathcal{R}, \tilde{\mu})$ such that $\left\|f-1_{\Delta}\right\|_{L^{2}(\mathcal{R}, \tilde{\mu})} \leq 1 / 2$ and $f$ is $\pi_{i, j}\left(\mathbb{Z}^{2}\right)$-invariant, i.e.

$$
f(x, y)=\eta_{a}^{i}(x) \overline{\eta_{a}^{j}(y)} f(x, y), \quad \forall a \in \mathbb{Z}^{2}
$$

$\tilde{\mu}$ almost everywhere $(x, y) \in \mathcal{R}$. 
Define

$$
S=\left\{(x, y) \in \mathcal{R} \mid \eta_{a}^{i}(x)=\eta_{a}^{j}(y), \forall a \in \mathbb{Z}^{2}\right\} .
$$

Then $S \subset \mathcal{R}$ is measurable and since $f \neq 0$, (3) implies that $\tilde{\mu}(S)>0$. We claim that for almost every $x \in X$, there is at most one $y \in X$ such that $(x, y) \in S$. If not, then we can find $X_{0} \subset X$ a set of positive measure and $\gamma \neq \gamma^{\prime} \in \Gamma$ such that

$$
\left(x, \sigma_{j}(\gamma)(x)\right),\left(x, \sigma_{j}\left(\gamma^{\prime}\right)(x)\right) \in S, \quad \forall x \in X_{0} .
$$

Thus, in particular, we get that

$$
\eta_{a}^{j}\left(\sigma_{j}(\gamma)(x)\right)=\eta_{a}^{j}\left(\sigma_{j}\left(\gamma^{\prime}\right)(x)\right), \quad \forall x \in X_{0}, \forall a \in \mathbb{Z}^{2},
$$

or, equivalently,

$$
a\left(p_{j}\left(\sigma_{j}(\gamma)(x)\right)\right)=a\left(p_{j}\left(\sigma_{j}\left(\gamma^{\prime}\right)(x)\right)\right), \quad \forall a \in \mathbb{Z}^{2}, \forall x \in X_{0} .
$$

Since characters separate points, we deduce that $p_{j}\left(\sigma_{j}(\gamma)(x)\right)=$ $p_{j}\left(\sigma_{j}\left(\gamma^{\prime}\right)(x)\right)$, for all $x \in X_{0}$. However, since $X_{0}$ is assumed to have positive measure, this contradicts condition (ii), thus proving the claim. Now, define $X_{i}$ to be the set of $x \in X$ with the property that there exists a unique $y \in X$ such that $(x, y) \in S$. The above claim and the fact that $\tilde{\mu}(S)>0$ imply that $\mu\left(X_{i}\right)>0$.

If $(x, y) \in S$, then $\eta_{a}^{i}(x)=\eta_{a}^{j}(y)$, for all $a \in \mathbb{Z}^{2}$, thus

$$
\eta_{\gamma(a)}^{i}(x)=\eta_{\gamma(a)}^{j}(y), \quad \forall a \in \mathbb{Z}^{2}, \forall \gamma \in \mathbb{F}_{2} .
$$

Since $\eta_{\gamma(a)}^{i}=\eta_{a}^{i} \circ \sigma_{i}\left(\gamma^{-1}\right)$, for all $a \in \mathbb{Z}^{2}$ and $\gamma \in \mathbb{F}_{2}$, we deduce that

$$
\left(\sigma_{i}(\gamma)(x), \sigma_{j}(\gamma)(y)\right) \in S, \quad \forall \gamma \in \mathbb{F}_{2} .
$$

In particular, we get that $X_{i}$ is $\sigma_{i}\left(\mathbb{F}_{2}\right)$-invariant. If we denote $X_{j}=\{y \in$ $\left.X \mid \exists x \in X_{i},(x, y) \in S\right\}$, then $X_{j}$ is a measurable $\sigma_{j}\left(\mathbb{F}_{2}\right)$-invariant set. Define $\phi: X_{i} \rightarrow X_{j}$ by $y=\phi(x)$ iff $(x, y) \in S$. Then $\phi$ is a measure preserving isomorphism. Indeed, as above, it follows that for almost every $y \in X$, there exists at most one $x \in X$ such that $(x, y) \in S$, hence $\phi$ is an isomorphism. Moreover, since $\phi(x)$ lies in the orbit of $x$ for almost every $x \in X$, we get that $\phi$ is measure preserving.

Finally, note that relation (4) implies that $\sigma_{j}(\gamma)(\phi(x))=\phi\left(\sigma_{i}(\gamma)(x)\right)$ almost everywhere $x \in X_{i}$ and for all $\gamma \in \mathbb{F}_{2}$, which gives the desired conjugacy.

Note that up to this point we have no examples of class $\mathcal{F}$ actions. This will be done in the next section by using a co-inducing construction for actions. 


\section{The co-induced action}

Let $\Gamma_{0} \subset \Gamma$ be two countable groups and let $\Gamma_{0} \curvearrowright^{\alpha}(Y, v)$ be a m.p. action. Then there is a natural way to construct a m.p. action of $\Gamma$ whose restriction to $\Gamma_{0}$ admits $\alpha$ as a quotient. We initially learned of this construction from Sect. 3.4 in [14], but it has been known for a while, being used for example in $[7,24]$. Start by defining

$$
X=\left\{f: \Gamma \rightarrow Y \mid f\left(\gamma \gamma_{0}\right)=\alpha\left(\gamma_{0}\right)(f(\gamma)), \forall \gamma_{0} \in \Gamma_{0}, \forall \gamma \in \Gamma\right\}
$$

and note that $\Gamma$ acts on $X$ by the formula $(\gamma f)\left(\gamma^{\prime}\right)=f\left(\gamma^{-1} \gamma^{\prime}\right)$, for all $\gamma$ and $\gamma^{\prime} \in \Gamma$.

Let $e \in S \subset \Gamma$ be a set such that $\Gamma=\sqcup_{s \in S} S \Gamma_{0}$. We observe that $X$ can be identified with $Y^{S}=\prod_{s \in S} Y$ via $f \rightarrow(f(s))_{s \in S}$. Using this identification we get an action $\tilde{\alpha}$ (called the co-induced action) of $\Gamma$ on $Y^{S}$ given by $\tilde{\alpha}(\gamma)\left(\left(x_{s}\right)_{s}\right)=\left(y_{s^{\prime}}\right)_{s^{\prime}}$, where $y_{s^{\prime}}=\alpha\left(\gamma_{0}^{-1}\right)\left(x_{s}\right)$ for the unique $s \in S$ and $\gamma_{0} \in \Gamma_{0}$ such that $\gamma^{-1} s^{\prime}=s \gamma_{0}$. Then $\tilde{\alpha}$ preserves the product measure $v_{S}=\bigotimes_{s \in S} v$ on $Y^{S}$.

In the next two lemmas we discuss the freeness and ergodicity of $\tilde{\alpha}$. Before this, we remark that $p: Y^{S} \rightarrow Y$ given by $p\left(\left(x_{s}\right)_{s}\right)=x_{e}$ is a quotient map and that $p$ realizes $\alpha$ as a quotient of $\tilde{\alpha}_{\mid \Gamma_{0}}$.

Lemma 2.1 Assume that $\alpha$ is a free action and that $(Y, v)$ is a non-atomic probability space. Then the set $A_{\gamma}=\left\{x \in Y^{S} \mid p(\gamma x)=p(x)\right\}$ has zero measure, for all $\gamma \in \Gamma \backslash\{e\}$. In particular, $\tilde{\alpha}$ is free.

Proof Note that if $\gamma \in \Gamma_{0} \backslash\{e\}$, then $A_{\gamma}=\left\{x \in Y^{S} \mid \gamma x_{e}=x_{e}\right\}$, hence the freeness of $\alpha$ implies that $A_{\gamma}$ has measure zero. On the other hand, if $\gamma \in$ $\Gamma \backslash \Gamma_{0}$, let $s \in S \backslash\{e\}$ and $\gamma_{0} \in \Gamma_{0}$ such that $\gamma^{-1}=s \gamma_{0}$. Then $A_{\gamma}=\{x \in$ $\left.Y^{S} \mid x_{e}=\gamma_{0}^{-1} x_{s}\right\}$, and since $Y$ is non-atomic, we get that $v_{S}\left(A_{\gamma}\right)=0$.

Lemma 2.2 In the above setting, let $\Lambda \subset \Gamma$ be a subgroup. Then

(i) If $\left|\Gamma / \Gamma_{0}\right|=\infty$, then $\tilde{\alpha}$ is weakly mixing. If $\left|\Gamma / \Gamma_{0}\right|<\infty$, then $\tilde{\alpha}$ is weakly mixing iff $\alpha$ is weakly mixing.

(ii) $\tilde{\alpha}$ is mixing iff $\alpha$ is mixing.

(iii) $\tilde{\alpha}_{\mid \Lambda}$ is weakly mixing iff $\alpha_{\mid s \Lambda s^{-1} \cap \Gamma_{0}}$ is weakly mixing for any $s \in \Gamma$ such that $s \Lambda s^{-1} \cap \Gamma_{0} \subset s \Lambda s^{-1}$ is of finite index.

(iv) $\tilde{\alpha}_{\mid \Lambda}$ is mixing iff $\alpha_{\mid s \Lambda s^{-1} \cap \Gamma_{0}}$ is mixing for any $s \in \Gamma$.

Proof Since (i) and (ii) follow by applying (iii) and (iv) to $\Lambda=\Gamma$, we only need to prove (iii) and (iv).

(iii) Consider the action of $\Gamma$ on $S$ given by

$$
\gamma \cdot s^{\prime}=s \quad \Leftrightarrow \quad \gamma s^{\prime} \in s \Gamma_{0} .
$$


For every $t \in S$ and $\gamma \in \Lambda$, let $\beta_{t}$ be the m.p. action of $\Lambda$ on $\prod_{s \in \Lambda \cdot t}(Y, v)_{s}$ given by $\beta_{t}(\gamma)\left(\left(x_{s}\right)_{s}\right)=\left(y_{s^{\prime}}\right)_{s^{\prime}}$, where $y_{s^{\prime}}=\alpha\left(\gamma_{0}^{-1}\right)\left(x_{s}\right)$, for the unique $s \in$ $\Lambda \cdot t$ and $\gamma_{0} \in \Gamma_{0}$ such that $\gamma^{-1} s^{\prime}=s \gamma_{0}$. Note that if $T \subset S$ is such that $S=\bigsqcup_{t \in T} \Lambda \cdot t$, then $\tilde{\alpha}_{\mid \Lambda}$ is the diagonal product of the actions $\beta_{t}$ with $t \in T$, i.e.

$$
\tilde{\alpha}_{\mid \Lambda}=\times_{t \in T} \beta_{t} .
$$

Claim 1 If $t \in T$ and $\Lambda \cdot t$ is infinite, then $\beta_{t}$ is weakly mixing.

Proof To prove that $\beta_{t}$ is weakly mixing we need to show that if $\xi_{1}, \ldots, \xi_{n} \in$ $L^{2}\left(\prod_{s \in \Lambda \cdot t}(Y, v)_{s}\right)$ are functions of zero integral, then for every $\varepsilon>0$ we can find $\gamma \in \Lambda$ such that $\left|\left\langle\beta_{t}(\gamma)\left(\xi_{i}\right), \xi_{j}\right\rangle\right| \leq \varepsilon$ for all $i, j$. Note that in order to prove this condition, we can assume that there exists a finite set $F \subset \Lambda \cdot t$ such that $\xi_{i} \in L^{2}\left(\prod_{s \in F}(Y, v)_{s}\right)$ for all $i=1, \ldots, n$.

Now, since $\Lambda \cdot t$ is infinite, we can find $\gamma \in \Lambda$ such that $\gamma F \cap F=\emptyset$. This implies that $\beta_{t}(\gamma)\left(\xi_{i}\right)$ and $\xi_{j}$ are independent for all $i, j$. Thus, $\left\langle\beta_{t}(\gamma)\left(\xi_{i}\right), \xi_{j}\right\rangle=0$ for all $i, j$, hence $\beta_{t}$ is weakly mixing.

Using Claim 1 we get that $\tilde{\alpha}_{\mid \Lambda}$ is weakly mixing iff $\beta_{t}$ is weakly mixing for every $t \in T$ such that $\Lambda \cdot t$ is finite. Let $t \in T$ such that $\Lambda \cdot t$ is finite. Then

$$
\Lambda_{t}=\left\{\gamma \in \Lambda \mid \gamma \cdot t^{\prime}=t^{\prime}, \forall t^{\prime} \in \Lambda \cdot t\right\}
$$

is a finite index subgroup of $\Lambda$. Thus, $\beta_{t}$ is weakly mixing iff $\beta_{t \mid \Lambda_{t}}$ is weakly mixing. Since

$$
\beta_{t}(\gamma)=\times_{s \in \Lambda \cdot t} \alpha\left(s^{-1} \gamma s\right), \quad \forall \gamma \in \Lambda_{t},
$$

we further deduce that $\beta_{t}$ is weakly mixing iff $\alpha_{\mid s^{-1} \Lambda_{t} s}$ is weakly mixing for every $s \in \Lambda \cdot t$. Next, note that the inclusions

$$
s^{-1} \Lambda_{t} s \subset s^{-1} \Lambda s \cap \Gamma_{0} \subset s^{-1} \Lambda s
$$

are of finite index for every $s \in \Lambda \cdot t$. This implies that $\beta_{t}$ is weakly mixing iff $\alpha_{\mid s^{-1} \Lambda s \cap \Gamma_{0}}$ is weakly mixing for every $s \in \Lambda \cdot t$. Altogether, we get that $\tilde{\alpha}_{\mid \Lambda}$ is weakly mixing iff $\alpha_{\mid s^{-1} \Lambda s \cap \Gamma_{0}}$ is weakly mixing for all $s \in \Gamma$ such that $\Lambda \cdot s$ is finite.

Since $\Lambda \cdot s$ is finite iff $\Lambda \cap s \Gamma_{0} s^{-1}=\{\gamma \in \Lambda \mid \gamma \cdot s=s\} \subset \Lambda$ is of finite index, we get the conclusion.

(iv) Assume that $\alpha_{\mid s \Lambda s^{-1} \cap \Gamma_{0}}$ is mixing for any $s \in \Gamma$. To prove that $\tilde{\alpha}_{\mid \Lambda}$ is mixing it suffices to show the following:

Claim 2 For any finitely supported vectors $f=\bigotimes_{s \in A} f_{s}, g=\bigotimes_{s \in B} g_{s} \in$ $L^{\infty}\left(Y^{S}, v_{S}\right)$, where $A, B \subset S$ are finite and $f_{s}, g_{t} \in L^{\infty}(Y, v)$ have zero in- 
tegral, for all $s \in A$ and $t \in B$, we have that

$$
\lim _{\Lambda \ni \gamma \rightarrow \infty}\langle\tilde{\alpha}(\gamma)(f), g\rangle=0 .
$$

Proof Note that the induced action $\tilde{\alpha}: \Gamma \rightarrow \operatorname{Aut}\left(L^{\infty}\left(Y^{S}\right)\right)$ is given by $\tilde{\alpha}(\gamma)\left(\bigotimes_{s} f_{s}\right)=\bigotimes_{s^{\prime}} g_{s^{\prime}}$, where $g_{s^{\prime}}=\alpha\left(\gamma_{0}\right)\left(f_{s}\right)$ for the unique $s \in S$ and $\gamma_{0} \in \Gamma_{0}$ such that $\gamma s=s^{\prime} \gamma_{0}$. Using this we get that if $\gamma \in \Gamma$, then $\langle\tilde{\alpha}(\gamma)(f), g\rangle=0$ unless $|A|=|B|$ and there exists a bijection $\pi: A \rightarrow B$ such that $\pi(s)^{-1} \gamma s \in \Gamma_{0}$ for all $s \in A$. In the latter case, we have that

$$
\left.\langle\tilde{\alpha}(\gamma)(f), g\rangle=\prod_{s \in A}\left\langle\alpha\left(\pi(s)^{-1} \gamma s\right)\right)\left(f_{s}\right), g_{\pi(s)}\right\rangle .
$$

For a bijection $\pi: A \rightarrow B$, let $\Lambda_{\pi}=\left\{\gamma \in \Lambda \mid \pi(s)^{-1} \gamma s \in \Gamma_{0}, \forall s \in A\right\}$. Then, proving the claim is equivalent to proving that

$$
\lim _{\Lambda_{\pi} \ni \gamma \rightarrow \infty}\langle\tilde{\alpha}(\gamma)(f), g\rangle=0,
$$

for all bijections $\pi: A \rightarrow B$. Fix a bijection $\pi: A \rightarrow B, \lambda \in \Lambda_{\pi}$ and $s \in A$. Then for all $\gamma \in \Lambda_{\pi}$ we have that $s^{-1}\left(\lambda^{-1} \gamma\right) s \in s^{-1} \Lambda s \cap \Gamma_{0}$ and that

$$
\begin{aligned}
\left.\left\langle\alpha\left(\pi(s)^{-1} \gamma s\right)\right)\left(f_{s}\right), g_{\pi(s)}\right\rangle & =\left\langle\alpha\left(\left(\pi(s)^{-1} \lambda s\right)\left(s^{-1}\left(\lambda^{-1} \gamma\right) s\right)\right)\left(f_{s}\right), g_{\pi(s)}\right\rangle \\
& =\left\langle\alpha\left(s^{-1}\left(\lambda^{-1} \gamma\right) s\right)\left(f_{s}\right), \alpha\left(s^{-1} \lambda^{-1} \pi(s)\right)\left(f_{\pi(s)}\right)\right\rangle .
\end{aligned}
$$

Now, if we let $\Lambda_{\pi} \ni \gamma \rightarrow \infty$, then $s^{-1} \Lambda s \cap \Gamma_{0} \ni s^{-1}\left(\lambda^{-1} \gamma\right) s \rightarrow \infty$. Since $\alpha_{\mid s^{-1} \Lambda s \cap \Gamma_{0}}$ is mixing by our assumption, we get that

$$
\left.\lim _{\Lambda_{\pi} \ni \gamma \rightarrow \infty}\left\langle\alpha\left(\pi(s)^{-1} \gamma s\right)\right)\left(f_{s}\right), g_{\pi(s)}\right\rangle=0,
$$

which ends the proof of the claim.

The other implication follows easily and we omit its proof.

For the next result, we assume the notations and assumptions of Sect. 1. Thus, $\Gamma$ is a countable group which contains $\Gamma_{0}=\mathbb{F}_{2}$ and $\alpha$ denotes the action $\mathbb{F}_{2} \curvearrowright \mathbb{T}^{2}$.

Corollary $2.3 \tilde{\alpha}$ is weakly mixing and belongs to $\mathcal{F}$. Moreover, for any ergodic action $\rho$ of $\Gamma$, the diagonal product action $\tilde{\alpha} \times \rho$ also belongs to $\mathcal{F}$.

Proof Since $\alpha$ is weakly mixing (see for example [31]), Lemma 2.2(ii) implies that $\tilde{\alpha}$ is weakly mixing. When combined with Lemma 2.1. this gives that $\tilde{\alpha} \in \mathcal{F}$. The second assertion follows easily since $\tilde{\alpha}$ is weakly mixing and thus the diagonal product with any ergodic action is still ergodic. 


\section{Proof of the Main Result}

Let $\Gamma$ be a countable group containing a fixed copy of $\mathbb{F}_{2}$. Let $\left\{\pi_{i}: \mathbb{F}_{2} \rightarrow\right.$ $\left.\mathcal{U}\left(\mathcal{H}_{i}\right)\right\}_{i \in I}$ be an uncountable family of mutually non-equivalent, irreducible, $\mathbf{c}_{0}$-representations of $\mathbb{F}_{2}$, i.e. such that $\lim _{g \rightarrow \infty}\left\langle\pi_{i}(g) \xi, \eta\right\rangle=0$ for all $\xi, \eta \in$ $\mathcal{H}_{i}$ and $i \in I$ [37].

Claim 1 For every $i \in I$, there exists a free, mixing, m.p. action $\Gamma \curvearrowright \rho_{i}$ $\left(X_{i}, \mu_{i}\right)$ such that

$$
\pi_{i} \subset \pi_{\rho_{i \mid \mathbb{F}_{2}}^{0}}^{0}
$$

Proof For every $i \in I$, let $\tilde{\pi}_{i}: \Gamma \rightarrow \mathcal{U}\left(\tilde{\mathcal{H}}_{i}\right)$ be the induced representation. Then we can find a free, m.p., Gaussian action $\Gamma \curvearrowright^{\rho_{i}}\left(X_{i}, \mu_{i}\right)$ (see for example $[5,13,22])$ such that

$$
\tilde{\pi}_{i} \subset \pi_{\rho_{i}}^{0} \subset \bigoplus_{n \geq 1} \tilde{\pi}_{i}^{\otimes_{n}}
$$

Now, since $\pi_{i}$ is $\mathbf{c}_{0}$, we get that $\tilde{\pi}_{i}$ is also $\mathbf{c}_{0}$, thus $\rho_{i}$ is a mixing action. Also, since $\pi_{i} \subset \tilde{\pi}_{i \mid \mathbb{F}_{2}}$ we get the second assertion.

Next, for every $i \in I$, consider the diagonal product action $\sigma_{i}=\tilde{\alpha} \times \rho_{i}$ of $\Gamma$ on

$$
\left(Z_{i}, \eta_{i}\right):=\prod_{s \in \Gamma / \mathbb{F}_{2}}\left(\mathbb{T}^{2}, \lambda^{2}\right)_{s} \times\left(X_{i}, \mu_{i}\right),
$$

where $\tilde{\alpha}$ denotes the action $\Gamma \curvearrowright \prod_{s \in \Gamma / \mathbb{F}_{2}}\left(\mathbb{T}^{2}, \lambda^{2}\right)_{s}$ obtained by co-inducing $\alpha$. Since $\tilde{\alpha}$ is free, weakly mixing and $\rho_{i}$ is mixing, we deduce that $\sigma_{i}$ is a free, ergodic action, for all $i \in I$.

Claim 2 Let $i \in I$ and let $Z_{i}^{\prime} \subset Z_{i}$ be a $\sigma_{i}\left(\mathbb{F}_{2}\right)$-invariant set of positive measure. Then the representation induced by the restriction of $\sigma_{i \mid \mathbb{F}_{2}}$ to $Z_{i}^{\prime}$ contains $\pi_{i}$.

Proof Since $\rho_{i}$ is mixing, we derive that $\rho_{i \mid \mathbb{F}_{2}}$ is weakly mixing. Thus, since $Z_{i}^{\prime}$ is $\sigma_{i}\left(\mathbb{F}_{2}\right)$-invariant, we get that $Z_{i}^{\prime}=B \times X_{i}$, for some measurable set $B \subset \prod_{s \in \Gamma / \mathbb{F}_{2}} \mathbb{T}^{2} \times Y$. This implies that the restriction of $\sigma_{i \mid \mathbb{F}_{2}}$ to $Z_{i}^{\prime}$ admits $\rho_{i \mid \mathbb{F}_{2}}$ as a quotient. Thus, the representation induced by the restriction of $\sigma_{i \mid \mathbb{F}_{2}}$ to $Z_{i}^{\prime}$ contains $\pi_{\rho_{i \mid \mathbb{F}_{2}}}^{0}$. Since, by Claim 1, the latter contains $\pi_{i}$, we are done.

Claim 3 For every $i \in I$, the set $I_{i}$ of $j \in I$ such that a restriction of $\sigma_{j \mid \mathbb{F}_{2}}$ is conjugate to a restriction of $\sigma_{i \mid \mathbb{F}_{2}}$ is countable. 
Proof Let $\pi$ be the unitary representation of $\mathbb{F}_{2}$ induced by $\sigma_{i \mid \mathbb{F}_{2}}$. If $j \in I_{i}$, then $\pi$ contains the representation induced by a restriction of $\sigma_{j_{\mid \mathbb{F}_{2}}}$. Now, by Claim 2, the latter contains $\pi_{j}$ as a subrepresentation. Combining these two inclusions, we get that $\pi_{j} \subset \pi$, for all $j \in I_{i}$. Since a separable unitary representation can only have countably many non-equivalent irreducible subrepresentations and since the $\pi_{j}^{\prime} s$ are irreducible and mutually non-equivalent, it follows that $I_{i}$ is countable.

Claim 4 Continuum many of the actions $\left\{\sigma_{i}\right\}_{i \in I}$ are mutually non-OE.

Proof If we assume the contrary, then we can find an uncountable set $J \subset I$ such that the actions $\left\{\sigma_{j}\right\}_{j \in J}$ are mutually orbit equivalent. Now, since $\beta \times \rho_{i}$ is ergodic, Corollary 2.3. implies that $\sigma_{i} \in \mathcal{F}$, for all $i \in I$. Thus, by applying Theorem 1.3. to the family of actions $\left\{\sigma_{j}\right\}_{j \in J} \subset \mathcal{F}$, we can find an uncountable subset $K \subset J$ such that for all $k, l \in K$, a restriction of $\sigma_{k \mid \mathbb{F}_{2}}$ is conjugate to a restriction of $\sigma_{l \mid \mathbb{F}_{2}}$. This, however, implies that $I_{k}$ is uncountable, for every $k \in K$, in contradiction with Claim 3. This finishes the proof of our main result.

Let us show moreover that if $\Gamma$ is a group containing $\mathbb{F}_{2}$ and if $\Lambda$ is measure equivalent to $\Gamma$, then $\Lambda$ admits a continuum of free, ergodic, non-OE actions. To this end, we first recall the definition of measure equivalence (see [12]). Let $\Gamma \curvearrowright^{\beta}(Y, \mu)$ be a free, ergodic, m.p. action and let $t>0$. Let $n>t$ be a natural number and set $Y^{n}=Y \times\{1, \ldots, n\}$ endowed with the natural measure. Next, let $Y^{t} \subset Y^{n}$ be a measurable set of measure $t$ and define $\mathcal{R}_{\beta}^{t}$ be the equivalence relation on $Y^{t}$ given by: $(x, i) \sim(y, j)$ iff there exists $\gamma \in \Gamma$ such that $y=\beta(\gamma)(x)$. Note that the isomorphism class of $\mathcal{R}_{\beta}^{t}$ depends on $t$ but not on the particular choice of $Y^{t}$ (since $\beta$ is ergodic). If $t=1$, then we use the notation $\mathcal{R}_{\beta}$. Two groups $\Gamma$ and $\Lambda$ are measure equivalent $(M E)$ if we can find an action $\beta$ as above, $t>0$ and a free, ergodic, m.p. action $\delta$ of $\Lambda$ on $Y^{t}$ such that

$$
\mathcal{R}_{\beta}^{t}=\mathcal{R}_{\delta} .
$$

Now, let $\Gamma \curvearrowright^{\theta}(S, m)$ be a weakly mixing, m.p. action. Then the diagonal product action $\theta \times \beta$ is ergodic and the equivalence relation $\mathcal{R}_{\theta \times \beta}^{t}$ can be realized as the equivalence relation on $S \times Y^{t}$ given by: $\left(s,((x, i)) \sim\left(s^{\prime},(y, j)\right)\right.$ iff there exists $\gamma \in \Gamma$ such that $s^{\prime}=\theta(\gamma)(s)$ and $y=\beta(\gamma)(x)$. Next, we note the following claim due to Gaboriau [14]:

Claim 5 In the context from above, there exists a free, ergodic, m.p. action $\tau$ of $\Lambda$ on $S \times Y^{t}$ such that

$$
\mathcal{R}_{\theta \times \beta}^{t}=\mathcal{R}_{\tau}
$$


Proof Let $\lambda \in \Lambda$, then for almost every $(x, i) \in Y^{t}$ we can find a unique (by the freeness of $\beta) \gamma=w(\lambda,(x, i)) \in \Gamma$ such that $\delta(\lambda)(x, i)=(\beta(\gamma)(x), i)$. Then $w: \Lambda \times Y^{t} \rightarrow \Gamma$ gives a cocycle for $\delta$. This implies that the formula

$$
\tau(\lambda)(s,(x, i))=(\theta(w(\lambda,(x, i)))(s), \delta(\lambda)(x, i))
$$

for all $\lambda \in \Lambda, s \in S,(x, i) \in Y^{t}$ defines a m.p. $\Lambda$-action on $S \times Y^{t}$. Moreover, it is clear that $\mathcal{R}_{\theta \times \beta}^{t}=\mathcal{R}_{\tau}$, hence, since $\theta \times \beta$ is ergodic, we get that $\tau$ is also ergodic. Also, since $\tau$ admits $\delta$ as a quotient and since $\delta$ is free, we deduce that $\tau$ is free.

Finally, let $\beta$ (resp. $\delta$ ) be a free, ergodic, m.p. action of $\Gamma$ (resp. of $\Lambda$ ) such that $\mathcal{R}_{\beta}^{t}=\mathcal{R}_{\delta}$, for some $t>0$. For all $i \in I$, denote $\theta_{i}=\tilde{\alpha} \times \rho_{i}$ and $\sigma_{i}=\theta_{i} \times \beta$. From the proofs of Claims 1-4 it follows that continuum many of the actions $\left\{\sigma_{i}\right\}_{i \in I}$ are non-orbit equivalent. On the other hand, by applying Claim 5, we get that for every $i \in I$ there exists a free, ergodic, m.p. action $\tau_{i}$ of $\Lambda$ such that $\mathcal{R}_{\sigma_{i}}^{t}=\mathcal{R}_{\tau_{i}}$. Recall that two actions are orbit equivalent iff they generate isomorphic equivalence relations. Thus, continuum many of the actions $\left\{\tau_{i}\right\}_{i \in I}$ are mutually non-orbit equivalent. This proves our moreover assertion.

\section{Applications to von Neumann algebras}

(I) After the first draft of this paper was posted on the arxiv (January 2007), there have been two important developments, in $[9,16]$. To briefly present these results, recall first that, in general, a non-amenable group $\Gamma$ need not contain a copy of $\mathbb{F}_{2}$ [28]. Nevertheless, D. Gaboriau and R. Lyons proved in [16] that any non-amenable group $\Gamma$ admits $\mathbb{F}_{2}$ as a measurable subgroup:

Theorem 4.1 [16] Let $\Gamma$ be a countable non-amenable group. Then there exist free, ergodic, m.p. actions $\Gamma \curvearrowright(Z, \eta)$ and $\mathbb{F}_{2} \curvearrowright(Z, \eta)$ such that $\mathbb{F}_{2} z \subset$ $\Gamma z$, a.e. $z \in Z$.

This result opened up the possibility that the condition $\Gamma$ contains a copy of $\mathbb{F}_{2}$ in the statement of our main theorem could be replaced by the more general, natural condition $\Gamma$ is non-amenable. To do this, by analogy with the proof of our main result, a co-inducing construction in a group/measurable subgroup situation rather than in a group/subgroup one, was needed.

Recently, I. Epstein obtained such a construction in [9] (see Lemma 4.2). Using this construction, she was able to push our arguments in the case that $\Gamma$ is an arbitrary non-amenable group and to show that indeed any such $\Gamma$ admits continuum many non-OE actions [9]. 
Lemma 4.2 [9] Let $\Gamma_{0}, \Gamma$ be two countable groups and assume that there exist free, ergodic, m.p. actions $\Gamma \curvearrowright(Z, \eta)$ and $\Gamma_{0} \curvearrowright(Z, \eta)$ such that $\Gamma_{0} z \subset \Gamma z$, a.e. $z \in Z$. Let $\Gamma_{0} \curvearrowright^{\alpha}(Y, v)$ be a free, ergodic, m.p. action. Then there exist a probability space $(X, \mu)$, a quotient map $p_{\alpha}: X \rightarrow Y$ and free, ergodic, m.p. actions $\Gamma_{0} \curvearrowright^{\beta}(X, \mu), \Gamma \curvearrowright^{\tilde{\alpha}}(X, \mu)$ such that

(i) $\alpha$ as a quotient of $\beta$ with $p_{\alpha}$ as the quotient map.

(ii) $\forall \gamma \in \Gamma \backslash\{e\}$, the set $\left\{x \in X \mid p_{\alpha}(\gamma x)=p_{\alpha}(x)\right\}$ has zero measure.

(iii) $\Gamma_{0} x \subset \Gamma x$, a.e. $x \in X$.

Below, we obtain some consequences of Theorem 4.1 and Lemma 4.2. in the theory of von Neumann algebras. We note that in the first draft of this paper, we obtained these corollaries under the additional assumption that $\Gamma$ contains a copy of $\mathbb{F}_{2}$.

(II) We begin by observing that one can characterize the non-amenability of a group $\Gamma$ in terms of Popa's notion of relative property (T) for von Neumann algebras. For this, let $M$ be a finite von Neumann algebra with a faithful, normal trace $\tau$ and let $B \subset M$ be a von Neumann subalgebra. The inclusion $(B \subset M)$ is rigid (or has relative property $(T)$ ) if whenever $\phi_{n}: M \rightarrow M$ is a sequence of unital, tracial, completely positive (c.p.) maps such that $\phi_{n} \rightarrow \mathrm{id}_{M}$ in the pointwise $\|\cdot\|_{2}$-topology, we must have that $\lim _{n \rightarrow \infty} \sup _{x \in B,\|x\| \leq 1}\left\|\phi_{n}(x)-x\right\|_{2}=0$ [30]. In the case $(B \subset M)=$ $\left(L\left(\Gamma_{0}\right) \subset L(\Gamma)\right)$, for two countable groups $\Gamma_{0} \subset \Gamma$, the inclusion $(B \subset M)$ is rigid iff the pair $\left(\Gamma, \Gamma_{0}\right)$ has relative property $(\mathrm{T})$ [30].

Also, recall that the group measure space construction associates to every free, ergodic, m.p. action $\Gamma \curvearrowright^{\sigma}(X, \mu)$ a $\mathrm{II}_{1}$ factor, $L^{\infty}(X, \mu) \rtimes_{\sigma} \Gamma$, together with a Cartan subalgebra, $L^{\infty}(X, \mu)$ [27]. In [30], Popa asked to characterize the countable groups $\Gamma$ which admit a rigid action $\sigma$, i.e. such that the inclusion $L^{\infty}(X, \mu) \subset L^{\infty}(X, \mu) \rtimes_{\sigma} \Gamma$ is rigid. The following result is motivated by this question.

Theorem 4.3 A countable group $\Gamma$ is non-amenable if and only if there exists a free, ergodic, m.p. action $\Gamma \curvearrowright(X, \mu)$ and a diffuse von Neumann subalgebra $Q \subset L^{\infty}(X, \mu)$ such that

(i) $Q^{\prime} \cap L^{\infty}(X, \mu) \rtimes \Gamma=L^{\infty}(X, \mu)$ and

(ii) the inclusion $Q \subset L^{\infty}(X, \mu) \rtimes \Gamma$ is rigid.

Proof If $\Gamma$ is amenable, then $L^{\infty}(X, \mu) \rtimes \Gamma$ is isomorphic to the hyperfinite $\mathrm{II}_{1}$ factor, $R$, for any free, ergodic, m.p. action $\Gamma \curvearrowright(X, \mu)[3,29]$. Since $R$ does not contain any diffuse von Neumann subalgebra with the relative property (T), we get the "if" part of the conclusion.

For the converse, let $\Gamma$ be a non-amenable group. As before, denote by $\alpha$ the action $\mathbb{F}_{2} \curvearrowright\left(\mathbb{T}^{2}, \lambda^{2}\right)$. Then, by combining Theorem 4.1 and Lemma 4.2 
we can find a probability space $(X, \mu)$, a quotient map $p: X \rightarrow Y=\mathbb{T}^{2}$ and two free, ergodic, m.p. actions $\mathbb{F}_{2} \curvearrowright^{\beta}(X, \mu), \Gamma \curvearrowright^{\tilde{\alpha}}(X, \mu)$ which satisfy conditions (i)-(iii) in Lemma 4.2.

Denote by $\theta: L^{\infty}\left(\mathbb{T}^{2}, \lambda^{2}\right) \hookrightarrow L^{\infty}(X, \mu)$ the embedding given by $\theta(f)=$ $f \circ p$, for all $f \in L^{\infty}\left(\mathbb{T}^{2}, \lambda^{2}\right)$, and let $Q=\theta\left(L^{\infty}\left(\mathbb{T}^{2}, \lambda^{2}\right)\right)$. We claim that $\tilde{\alpha}$ and $Q$ verify the conclusion. For this, denote by $\left\{u_{\gamma}\right\}_{\gamma \in \Gamma}$ the canonical unitaries implementing the action of $\Gamma$ on $L^{\infty}(X, \mu)$. Then it is easy to see that $Q^{\prime} \cap L^{\infty}(X, \mu) \rtimes_{\tilde{\alpha}} \Gamma$ is generated by $L^{\infty}(X, \mu)$ and $\left\{1_{A_{\gamma}} u_{\gamma} \mid \gamma \in \Gamma\right\}$, where $A_{\gamma}=\left\{x \in X \mid p\left(\gamma^{-1} x\right)=p(x)\right\}$, for all $\gamma \in \Gamma$. As $\mu\left(A_{\gamma}\right)=0$, for all $\gamma \in \Gamma \backslash\{e\}$, we deduce that $Q^{\prime} \cap L^{\infty}(X, \mu) \rtimes_{\tilde{\alpha}} \Gamma=L^{\infty}(X, \mu)$.

Next, since $p$ realizes $\alpha$ as a quotient of $\beta$, we get that $\theta$ extends to an embedding

$$
\theta: L^{\infty}\left(\mathbb{T}^{2}, \lambda^{2}\right) \rtimes_{\alpha} \mathbb{F}_{2} \hookrightarrow L^{\infty}(X, \mu) \rtimes_{\beta} \mathbb{F}_{2} .
$$

Now, by [30], the inclusion $L^{\infty}\left(\mathbb{T}^{2}, \lambda^{2}\right) \subset L^{\infty}\left(\mathbb{T}^{2}, \lambda^{2}\right) \rtimes_{\alpha} \mathbb{F}_{2}$ is rigid, hence the inclusion $Q \subset L^{\infty}(X, \mu) \rtimes_{\beta} \mathbb{F}_{2}$ is rigid. Finally, since $\mathbb{F}_{2} x \subset \Gamma x$, a.e. $x \in X$, we have that $L^{\infty}(X, \mu) \rtimes_{\beta} \mathbb{F}_{2} \subset L^{\infty}(X, \mu) \rtimes_{\tilde{\alpha}} \Gamma$ and we deduce that the inclusion $Q \subset L^{\infty}(X, \mu) \rtimes_{\tilde{\alpha}} \Gamma$ is rigid [30].

Remark 4.4 Theorem 4.3 implies that every countable non-amenable group $\Gamma$ admits an almost rigid action. To make this precise, let $\sigma$ be the action given by Theorem 4.3 and let $\left\{p_{n}\right\}_{n \geq 1}$ be a sequence of projections which generate $L^{\infty}(X, \mu)$. For every $n$, define $Q_{n}=\left(Q \vee\left\{p_{1}, \ldots, p_{n}\right\}\right)^{\prime \prime}$. Then the inclusion $Q_{n} \subset L^{\infty}(X, \mu) \rtimes_{\sigma} \Gamma$ is rigid and $Q_{n}^{\prime} \cap L^{\infty}(X, \mu) \rtimes_{\sigma} \Gamma=L^{\infty}(X, \mu)$, for all $n$. Moreover, we have that $\overline{\bigcup_{n \geq 1} Q_{n}} w=L^{\infty}(X, \mu)$.

Next, we denote by $A=\bigoplus_{1}^{\infty} \mathbb{Z}$ and we note that if $\Gamma$ contains s copy $\mathbb{F}_{2}$ then the action $\sigma$ from Theorem 4.3 can be taken to come from an action of $\Gamma$ by automorphisms on $A$.

Proposition 4.5 Let $\Gamma$ be a countable group which contains $\mathbb{F}_{2}$. Then there exists a homomorphism $\rho: \Gamma \rightarrow \operatorname{Aut}(A)$ and an infinite subgroup $B \subset A$ such that the pair $\left(\Gamma \ltimes_{\rho} A, B\right)$ has relative property $(T)$ and that the set $\left\{\gamma(b) b^{-1} \mid b \in B\right\}$ is infinite, for all $\gamma \in \Gamma \backslash\{e\}$.

Proof First, remark that $\mathbb{F}_{2}$ contains a copy of itself which has infinite index. Indeed, if $\mathbb{F}_{2}=\langle a, b\rangle$, then the subgroup generated by $a$ and $b a b^{-1}$ has infinite index and is isomorphic to $\mathbb{F}_{2}$. Thus, we can assume that $\mathbb{F}_{2}$ has infinite index in $\Gamma$.

Next, let $e \in S \subset \Gamma$ be a set such that $\Gamma=\bigsqcup_{s \in S} s \mathbb{F}_{2}$ and identify $A$ with $\bigoplus_{s \in S} \mathbb{Z}^{2}$. Then the co-induced construction from Sect. 2 shows the action $\alpha: \mathbb{F}_{2} \rightarrow \operatorname{Aut}\left(\mathbb{Z}^{2}\right)$ co-induces to an action $\rho: \Gamma \rightarrow \operatorname{Aut}(A)$. Moreover, we have that $\rho\left(\mathbb{F}_{2}\right)$ invaries $B=\left(\mathbb{Z}^{2}\right)_{e}$ and that the inclusions of groups $(B \subset$ 
$\left.\mathbb{F}_{2} \ltimes_{\rho_{\mid \mathbb{F}_{2}}} B\right)$ and $\left(\mathbb{Z}^{2} \subset \mathbb{F}_{2} \ltimes_{\alpha} \mathbb{Z}^{2}\right)$ are isomorphic. Since the latter inclusion has relative property $(\mathrm{T})$, we deduce that the pair $\left(\Gamma \ltimes_{\rho} A, B\right)$ has relative property $(\mathrm{T})$. The second assertion is easy and we leave it to the reader.

In connection with the statements of Theorem 4.3 and Proposition 4.5, note that $\mathrm{T}$. Férnos proved that a countable group $\Gamma$ can act on a non-trivial Abelian group $A$ of finite $\mathbb{Q}$-rank such that the pair $(\Gamma \rtimes A, A)$ has relative property $(\mathrm{T})$ if and only if it admits a linear representation $\phi: \Gamma \rightarrow \mathrm{SL}_{n}(\mathbb{R})$ with non-amenable Zariski closure, $\overline{\phi(\Gamma)}[10]$.

In the context of Proposition 4.5, it now follows that the induced action $\Gamma \curvearrowright^{\sigma}(\hat{A}, \mu)$ verifies Theorem 4.3, where $\mu$ is the Haar measure on the dual of $A$. We note that we do not know whether the converse of Proposition 4.5 is true, i.e. if any countable group $\Gamma$ which has an action on $A$ with the above properties must necessarily contain $\mathbb{F}_{2}$.

The class of $\mathcal{H} \mathcal{T}$ factors has been introduced by Popa, who used it to provide the first examples of $\mathrm{II}_{1}$ factors with trivial fundamental group [30]. A $\mathrm{II}_{1}$ factor $M$ is in the $\mathcal{H} \mathcal{T}$ class if it has a Cartan subalgebra $A$ (called an HT Cartan subalgebra) such that:

(i) $M$ has the property $\mathrm{H}$ relative to $A$ and

(ii) there exists a von Neumann subalgebra $B \subset A$ such that $B^{\prime} \cap M \subset A$ and the inclusion $B \subset M$ is rigid.

In [30], Popa raised the question of characterizing HT groups, i.e. groups which admit a free, ergodic, m.p. action $\Gamma \curvearrowright^{\sigma}(X, \mu)$ such that the corresponding Cartan subalgebra inclusion $(A \subset M)=\left(L^{\infty}(X, \mu) \subset\right.$ $\left.L^{\infty}(X, \mu) \rtimes_{\sigma} \Gamma\right)$ is HT. Since in this case, $M$ has property $\mathrm{H}$ relative to $A$ if and only if $\Gamma$ has Haagerup's property [30], Theorem 4.3 implies the following:

Corollary 4.6 A countable group $\Gamma$ is $H T$ if and only if is non-amenable and has Haagerup's property.

(III) Recall that two actions $\Gamma \curvearrowright(X, \mu)$ and $\Lambda \curvearrowright(Y, v)$ are called von Neumann equivalent $(v N E)$ if the associated $\mathrm{II}_{1}$ factors are isomorphic, i.e. if $L^{\infty}(X, \mu) \rtimes \Gamma \simeq L^{\infty}(Y, v) \rtimes \Lambda$ [32]. Next, we show that any non-amenable group admits continuum many non-von Neumann equivalent actions. Since von Neumann equivalence of actions is weaker than orbit equivalence [11] this result generalizes Theorem 1.3 as well as the main result of [9]. For $\Gamma=\mathbb{F}_{n}, n \geq 2$, this result has been first obtained in [GP].

Theorem 4.7 Any countable, non-amenable group $\Gamma$ admits continuum many non-vNE free, ergodic m.p. actions. 
Proof Let $\tilde{\mathcal{F}}$ be the class of free, ergodic, m.p. actions $\Gamma \curvearrowright^{\sigma}(X, \mu)$ such that there exists a free, ergodic, m.p. action $\mathbb{F}_{2} \curvearrowright^{\beta}(X, \mu)$ satisfying the following

(i) $\alpha$ is a quotient of $\beta$, with the quotient map $p_{\sigma}: X \rightarrow \mathbb{T}^{2}$,

(ii) $\forall \gamma \in \Gamma \backslash\{e\}$, the set $\left\{x \in X \mid p_{\sigma}(\gamma x)=p_{\sigma}(x)\right\}$ has zero measure and

(iii) $\mathbb{F}_{2} x \subset \Gamma x$, a.e. $x \in X$.

It is then proven in [9], by using Theorem 1.3, that there exists an uncountable family of actions $\Gamma \curvearrowright^{\sigma_{i}}\left(X_{i}, \mu_{i}\right)(i \in I)$ from $\tilde{\mathcal{F}}$ which are mutually non-orbit equivalent. For every $i \in I$, denote $M_{i}=L^{\infty}\left(X_{i}, \mu_{i}\right) \rtimes_{\sigma_{i}} \Gamma$ and $A_{i}=L^{\infty}\left(X_{i}, \mu_{i}\right)$.

Claim For every $i_{0} \in I$, the set $J=\left\{i \in I \mid M_{i} \simeq M_{i_{0}}\right\}$ is countable.

Note that since $I$ is uncountable, this claim implies that continuum many of the actions $\left\{\sigma_{i}\right\}_{i \in I}$ are non-von Neumann equivalent.

Proof Start by denoting $Q=L^{\infty}\left(\mathbb{T}^{2}, \lambda^{2}\right)$ and $N=L^{\infty}\left(\mathbb{T}^{2}, \lambda^{2}\right) \rtimes_{\alpha} \mathbb{F}_{2}$. Since $\sigma_{i} \in \tilde{\mathcal{F}}$, the proof of Theorem 4.3. shows that there exists an embedding of $N$ into $M_{i}$ such that under this embedding $Q \subset A_{i}$ and $Q^{\prime} \cap M_{i}=A_{i}$. Also, since the inclusion $Q \subset N$ is rigid [30], we can find $F \subset N$ finite and $\delta>0$ such that if a unital, tracial, c.p. map $\phi: N \rightarrow N$ satisfies $\|\phi(x)-x\|_{2} \leq \delta$, for all $x \in F$, then

$$
\|\phi(b)-b\|_{2} \leq 1 / 4, \quad \forall b \in(Q)_{1} .
$$

To prove the claim, assume by contradiction that $J$ is uncountable. For every $i \in J$, let $\theta_{i}: M_{i} \rightarrow M_{i_{0}}$ be an isomorphism and consider the set $\left\{\theta_{i}(x) \mid x \in F\right\} \subset L^{2}\left(M_{i_{0}}\right)^{\oplus|F|}$. Since $L^{2}\left(M_{i_{0}}\right)$ is a separable Hilbert space and since $J$ is uncountable, we can find $i \neq j \in J$ such that

$$
\left\|\theta_{i}(x)-\theta_{j}(x)\right\|_{2} \leq \delta, \quad \forall x \in F .
$$

Thus, the isomorphism $\theta=\theta_{j}^{-1} \circ \theta_{i}: M_{i} \rightarrow M_{j}$ satisfies $\|\theta(x)-x\|_{2} \leq \delta$, for all $x \in F$.

Further, if we let $\phi=\left(E_{N} \circ \theta\right)_{\mid N}: N \rightarrow N$ (where $E_{N}: M_{j} \rightarrow N$ is the conditional expectation onto $N$ ), then $\phi$ is a unital, tracial, c.p. map and

$$
\|\phi(x)-x\|_{2}=\left\|E_{N}(\theta(x))-x\right\|_{2}=\left\|E_{N}(\theta(x)-x)\right\|_{2} \leq \delta, \quad \forall x \in F .
$$

Using the fact that the inclusion $Q \subset N$ is rigid, (1) implies that

$$
\left\|E_{N}(\theta(u))-u\right\|_{2}=\|\phi(u)-u\|_{2} \leq 1 / 4, \quad \forall u \in \mathcal{U}(Q) .
$$


Since $Q \subset N$, (6) implies that

$$
\begin{aligned}
\| \theta & (u) u^{*}-1 \|_{2}^{2} \\
& =2-2 \Re \tau\left(\theta(u) u^{*}\right) \\
& =2-2 \Re \tau\left(E_{N}(\theta(u)) u^{*}\right)=2 \Re \tau\left(\left(u-E_{N}(\theta(u))\right) u^{*}\right) \\
& \leq 2\left\|u-E_{N}(\theta(u))\right\|_{2} \leq 1 / 2, \quad \forall u \in \mathcal{U}(Q) .
\end{aligned}
$$

Next, we use a standard averaging trick. For this, let $K$ denote the $\|\cdot\|_{2}$ closure of the convex hull of the set $\left\{\theta(u) u^{*} \mid u \in \mathcal{U}(Q)\right\}$ and let $\xi \in K$ be the element of minimal norm. Using (7) and the fact that $K \subset\left(M_{j}\right)_{1}$, we deduce that $\|\xi\| \leq 1$ and that $\|\xi-1\|_{2} \leq 1 / 2$, so, in particular, $\xi \neq 0$. Moreover, since $K$ is invariant under the $\|\cdot\|_{2}$-preserving transformations $K \ni \eta \rightarrow \theta(u) \eta u^{*}$, for all $u \in \mathcal{U}(Q)$, the uniqueness of $\xi$ implies that $\theta(u) \xi u^{*}=\xi$, for all $u \in \mathcal{U}(Q)$. Furthermore, it is easy to see that this relation is still verified if we replace $\xi$ by the partial isometry $v$ in its polar decomposition and the unitary $u \in Q$ by an arbitrary element $x \in Q$ (since any element in $\mathrm{C}^{*}$-algebra is a linear combination of 4 unitaries), i.e.

$$
\theta(x) v=v x, \quad \forall x \in Q .
$$

Using (8) it follows immediately that $v^{*} v \in Q^{\prime} \cap M_{j}$ and that $v v^{*} \in \theta(Q)^{\prime} \cap$ $M_{j}=\theta\left(Q^{\prime} \cap M_{i}\right)$. Denote $q=v v^{*}, p_{i}=\theta^{-1}(q)$ and $p_{j}=v^{*} v$. Since $Q^{\prime} \cap$ $M_{k}=A_{k}$, for every $k \in I$, we get that $p_{i} \in A_{i}$ and $p_{j} \in A_{j}$.

Now, if we define $\delta(x)=v^{*} \theta(x) v$, for all $x \in p_{i} M_{i} p_{i}$, then $\delta: p_{i} M_{i} p_{i} \rightarrow$ $p_{j} M_{j} p_{j}$ is an isomorphism. Moreover, (8) implies that for all $x \in Q$ we have that

$$
\delta\left(x p_{i}\right)=v^{*} \theta\left(x p_{i}\right) v=v^{*} \theta(x) v=v^{*} v x=x p_{j} .
$$

In particular, (9) implies that $\delta\left(\left(Q p_{i}\right)^{\prime} \cap p_{i} M_{i} p_{i}\right)=\left(Q p_{j}\right)^{\prime} \cap p_{j} M_{j} p_{j}$, or, equivalently, that $\delta\left(A_{i} p_{i}\right)=A_{j} p_{j}$. Altogether, we get that $\delta$ gives an isomorphism of the inclusions $\left(A p_{i} \subset p_{i} M_{i} p_{i}\right) \simeq\left(A p_{j} \subset p_{j} M_{j} p_{j}\right)$. Finally, since $p_{i}$ and $p_{j}$ have the same trace, we would get that $\left(A_{i} \subset M_{i}\right) \simeq\left(A_{j} \subset M_{j}\right)$, i.e. the actions $\sigma_{i}$ and $\sigma_{j}$ are orbit equivalent [11], a contradiction.

Acknowledgements I am grateful to Professors Damien Gaboriau, Alekos Kechris and Sorin Popa for useful discussions and helpful comments.

Open Access This article is distributed under the terms of the Creative Commons Attribution Noncommercial License which permits any noncommercial use, distribution, and reproduction in any medium, provided the original author(s) and source are credited. 


\section{References}

1. Bezuglyi, S.I., Golodets, V.Ya.: Hyperfinite and $\mathrm{II}_{1}$ actions for nonamenable groups. J. Funct. Anal. 40(1), 30-44 (1981)

2. Burger, M.: Kazhdan constants for SL(3, ZZ). J. Reine Angew. Math. 413, 36-67 (1991)

3. Connes, A.: Classification of injective factors. Cases $\mathrm{II}_{1}, \mathrm{II}_{\infty}, \mathrm{III}_{\lambda}, \lambda \neq 1$. Ann. Math. (2) 104(1), 73-115 (1976)

4. Connes, A.: A factor of type $\mathrm{II}_{1}$ with countable fundamental group. J. Oper. Theory 4(1), 151-153 (1980)

5. Connes, A., Weiss, B.: Property (T) and asymptotically invariant sequences. Isr. J. Math. 37, 209-210 (1980)

6. Connes, A., Feldman, J., Weiss, B.: An amenable equivalence relation is generated by a single transformation. Ergod. Theory Dyn. Syst. 1, 431-450 (1981)

7. Dooley, H., Golodets, V.Ya., Rudolph, D.J., Sinelø'shchikov, D.: Non-Bernoulli systems with completely positive entropy. Ergod. Theory Dyn. Syst. 28, 87-124 (2008)

8. Dye, H.: On groups of measure preserving transformations I. Am. J. Math. 81, 119-159 (1959)

9. Epstein, I.: Orbit inequivalent actions of non-amenable groups. arXiv:0707.4215 [math.GR]

10. Férnos, T.: Relative property (T) and linear groups. Ann. Inst. Fourier (Grenoble) 56(6), 1767-1804 (2006)

11. Feldman, J., Moore, C.C.: Ergodic equivalence relations, cohomology, and von Neumann algebras, II. Trans. Am. Math. Soc. 234, 325-359 (1977)

12. Furman, A.: Orbit equivalence rigidity. Ann. Math. (2) 150(3), 1083-1108 (1999)

13. Furman, A.: On Popa's cocycle superrigidity theorem. Int. Math. Res. Not. 19, rnm073 (2007), 46 pp.

14. Gaboriau, D.: Examples of groups that are measure equivalent to the free group. Ergod. Theory Dyn. Syst. 25(6), 1809-1827 (2005)

15. Gefter, S.L., Golodets, V.Y.: Fundamental groups for ergodic actions and actions with unit fundamental groups. Publ. Res. Inst. Math. Sci. 24(6), 821-847 (1988)

16. Gaboriau, D., Lyons, R.: A measurable-group-theoretic solution to von Neumann's problem. Invent. Math. 177, 533-540 (2009)

17. Gaboriau, D., Popa, S.: An uncountable family of non orbit equivalent actions of $\mathbb{F}_{n}$. J. Am. Math. Soc. 18, 547-559 (2005)

18. Hjorth, G.: A converse to Dye's theorem. Trans. Am. Math. Soc. 357, 3083-3103 (2005)

19. Ioana, A.: A relative version of Connes' $\chi(M)$ invariant and existence of orbit inequivalent actions. Ergod. Theory Dyn. Syst. 27(4), 1199-1213 (2007)

20. Ioana, A.: Non-orbit equivalent actions of $\mathbb{F}_{n}$. Ann. Sci. ENS 42, 675-696 (2009)

21. Kazhdan, D.: On the connection of the dual space of a group with the structure of its closed subgroups. Funct. Anal. Appl. 1, 63-65 (1967)

22. Kechris, A.: Global Aspects of Ergodic Group Actions and Equivalence Relations. Mathematical Surveys and Monographs, vol. 160. American Mathematical Society, Providence (2010)

23. Kida, Y.: Orbit equivalence rigidity for ergodic actions of the mapping class group. Geom. Dedic. 131, 99-109 (2008)

24. Lück, W.: The type of the classifying space for a family of subgroups. J. Pure Appl. Algebra 149(2), 177-203 (2000)

25. Margulis, G.: Finitely-additive invariant measures on Euclidian spaces. Ergod. Theory Dyn. Syst. 2, 383-396 (1982)

26. Monod, N., Shalom, Y.: Orbit equivalence rigidity and bounded cohomology. Ann. Math. 164, 825-878 (2006)

27. Murray, F., von Neumann, J.: Rings of operators, IV. Ann. Math. 44, 716-808 (1943) 
28. Ol'shanskii, A.Yu.: On the question of the existence of an invariant mean on a group. Usp. Mat. Nauk 35(4(214)), 199-200 (1980)

29. Ornstein, D., Weiss, B.: Ergodic theory of amenable groups. I. The Rokhlin lemma. Bull. Amer. Math. Soc. (N.S.) 1, 161-164 (1980)

30. Popa, S.: On a class of type $\mathrm{II}_{1}$ factors with Betti numbers invariants. Ann. Math. 163, 809-889 (2006)

31. Popa, S.: Some computations of 1-cohomology groups and construction of non-orbitequivalent actions. J. Inst. Math. Jussieu 5, 309-332 (2006)

32. Popa, S.: Strong rigidity of $\mathrm{II}_{1}$ factors arising from malleable actions of w-rigid groups II. Invent. Math. 165, 409-451 (2006)

33. Popa, S.: On the superrigidity of malleable actions with spectral gap. J. Am. Math. Soc. 21(4), 981-1000 (2008)

34. Shalom, Y.: Bounded generation and Kazhdan's property (T). Inst. Ht. Études Sci. Publ. Math. 90, 145-168 (1999)

35. Shalom, Y.: Measurable Group Theory. European Congress of Mathematics, pp. 391-423. Eur. Math. Soc., Zürich (2005)

36. Schmidt, K.: Amenability, Kazhdan's property T, strong ergodicity and invariant means for ergodic group-actions. Ergod. Theory Dyn. Syst. 1(2), 223-236 (1981)

37. Szwarc, R.: An analytic series of irreducible representations of the free group. Ann. Inst. Fourier 38, 87-110 (1988)

38. Törnquist, A.: Orbit equivalence and actions of $\mathbb{F}_{n}$. J. Symb. Log. 71(1), 265-282 (2006)

39. Zimmer, R.: Ergodic Theory and Semisimple Groups. Birkhauser, Boston (1984) 